\title{
Jogging and Walking Analysis Using Wearable Sensors*
}

\author{
Ching Yee Yong, Rubita Sudirman, Ahmad Hazwan Ab Rahim, \\ Nasrul Humaimi Mahmood, Kim Mey Chew \\ Infocomm Research Alliance, Faculty of Electrical Engineering, Universiti Teknologi Malaysia, Johor, Malaysia \\ Email: rubita@fke.utm.my
}

Received 2013

\begin{abstract}
Gait analysis is a process of learning the motion of human and animal by using wearable sensor approach and vision approach. This analysis is mainly used in medical and sports field where the study of body parts is crucial. 3-space sensor is a sensor consists of accelerometer, gyroscope sensor and compass sensor, built in one device. In this study, 3 -space sensor is used to collect data of walking and jogging motion, of a test subject running on a treadmill. Angular velocity of the test subject's arm and the angle of subject's leaping motion are the two main components under investigation. Data are analyzed and processed with Principal of Component Analysis (PCA) technique. This method aims to combine and reduce the number of variables of the raw data. The Quiver function is used in order to generate feature vectors for both motions. Furthermore, the output of the process was used to create a system that can recognize human motion on any given data. The system is highly able to differentiate both of the motions.
\end{abstract}

Keywords: Accelerometer; Gyroscope; Motion; Wearable Sensor; Leaping

\section{Introduction}

Gait analysis is a study about movement of animal, or in other words, a study of animal locomotion, with the aim to analyze human motions, which is widely used in medical purposes. This implementation is crucial to illustrate the different body dynamics which depend on the test subjects' conditions, as stated in [1].

This analysis can be done by using two methods. The first method, which is classified as vision based approach, using video camera to record the movement of the subject. The second method is using wearable sensor, by attaching a tri-axial sensor to the upper limb of the test subject to generate sets of motion data.

In this paper, the second method is implemented. 3 -space sensor is attached on the test subject to acquire the motion data. Graphs were generated to represent the orientations, rotation and acceleration (force) of the motion which are walking and jogging. A system is developed to recognize and differentiate these motions.

\section{Research Review}

Gait analysis is a very important study of human locomotive that give a great advantage in sports and medical purposes. Zhou et al. [2] used multiple wearable inertial sensors on upper limb motion tracking to give appropriate assessment to the stroke patients before they are dis-

"Motion classification using angular velocity and leaping angle. charged from the hospital after their movements are comparable to the normal movement of human. Other than that, Liu et al. [3] used wearable sensor approach in gait recognition experiment, where the movement of a subject was used to recognize that particular individual. This is one of the application using wearable sensors as a domain in biometric identification.

3-space sensor consists of 3 types of sensor, tri-axial accelerometer, tri-axial gyroscope and tri-axial compass which is built in one device [4] as shown in Figure 1. Some experiment needed three types of sensor to evaluate the entire motions component, but this device can generate data for all components simultaneously in one move by the subject.

Accelerometer sensor: this sensor can sense acceleration by gravitational force and the acceleration of moving body. Measured in g. $1 \mathrm{~g}$ equals to $9.81 \mathrm{~m} / \mathrm{s}^{2}$, which is the value of acceleration of gravitational force. Gyroscope

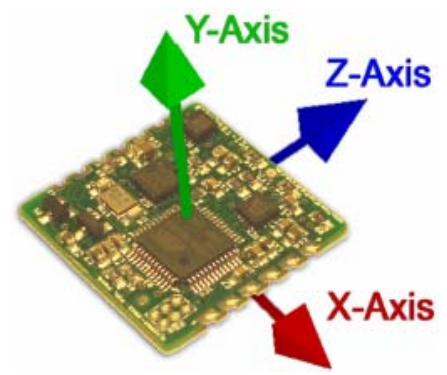

Figure 1. 3-space sensor axis orientations. 
sensor: this sensor measure angular velocity in $\mathrm{rad} / \mathrm{s}$. Compass: measures rotation angle with earth magnetic pole as references. Hence, any magnetic or metal object might disrupt the reading of this sensor, measured in degree.

\section{Materials and Methods}

\subsection{Data Collection}

A test subject was selected and his movement was evaluated thoroughly to differentiate the motion of a subject. The subject for this experiment is a healthy male individual, with the age of 23 , without medical record of defect or other sicknesses. The test subject was attached with the 3-space sensor on his upper arm, for jogging and walking assessment. These motions were performed on a controlled treadmill with wireless connection from the sensor to the wireless dongle. Treadmill was used as a medium for test subject to perform a regular motion with lowest bias.

A preliminary speed testing was done before collecting any data. The test subject needs to perform a normal walk and jog on ground for a distance of 10 meter. Time was stamped to record the period for the test subject to cover up the activities. Hence, the test subject's normal walking and jogging speed was identified with walking $3.7 \mathrm{feet} / \mathrm{second}$ and jogging $6.5 \mathrm{feet} / \mathrm{second}$. This information was used as speed setting of the treadmill.

For walking motion, the test subject was asked to perform a normal walking on the treadmill with speed of 3.7 feet/second for a 10 meter, 15 meter, 20 meter, 25 meter and 30 meter as a session. Then, every single session was repeated 10 times for each of the different distance. Jogging activity with speed 6.5 feet/second was performed using the same characteristics of the walking activity. Thus 50 sets of data for jogging and walking motion were logged by the sensor were saved in .txt file.

\subsection{Angular Velocity}

The leg and arm are playing important role in human motion. These two components are moving in a periodical wave while the test subject is performing jogging and walking activities. The arm of the subject is the main focus of the study.

While moving forward, the test subject is swinging his arm forward and backward (rotation in y axis) and performing rotation in vertical axis (z-axis), as shown in Figure 2. Rotation in $\mathrm{x}$-axis is very small for walking and jogging and hence data for this are ignored.

The main thing of the analysis is to detect the positive angular velocity for each swing of jogging and walking activities and then calculate the mean value of every swing. PCA is used to get the best view of the motion on the graph after the variables were reduced as mentioned in next subsection. Since the rotations of the arm during activities are not perfectly aligned along the $\mathrm{y}$ and $\mathrm{z}$-axis, PCA was used to realign the graph to get the actual axis of the moving arms rotations, as pictured in Figure 3.

\subsection{Principle Component Analysis (PCA)}

Principle of component analysis process shown in Figure 4 is a procedure in analyzing 2 or 3 dimensional set of data, or to minimize the number of data by creating an artificial point to represent the set of data. A randomly 100 points of data were selected from each session for PCA analysis. The scatter plot graph shown 100 points of distribution after PCA and each point was represented as 3 aspects (acceleration, angular motion and angle) for a data collecting event, as written in [5]. Several steps were performed such as covariance, covariance matrix, Eigen vector and Eigen value to generate an artificial point for a set of data for PCA variables reduction.

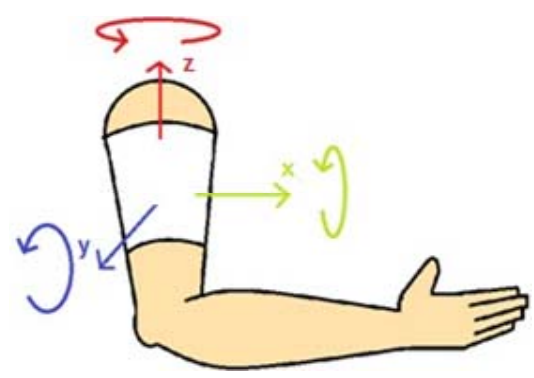

Figure 2. Rotation motion of test subject's arm.

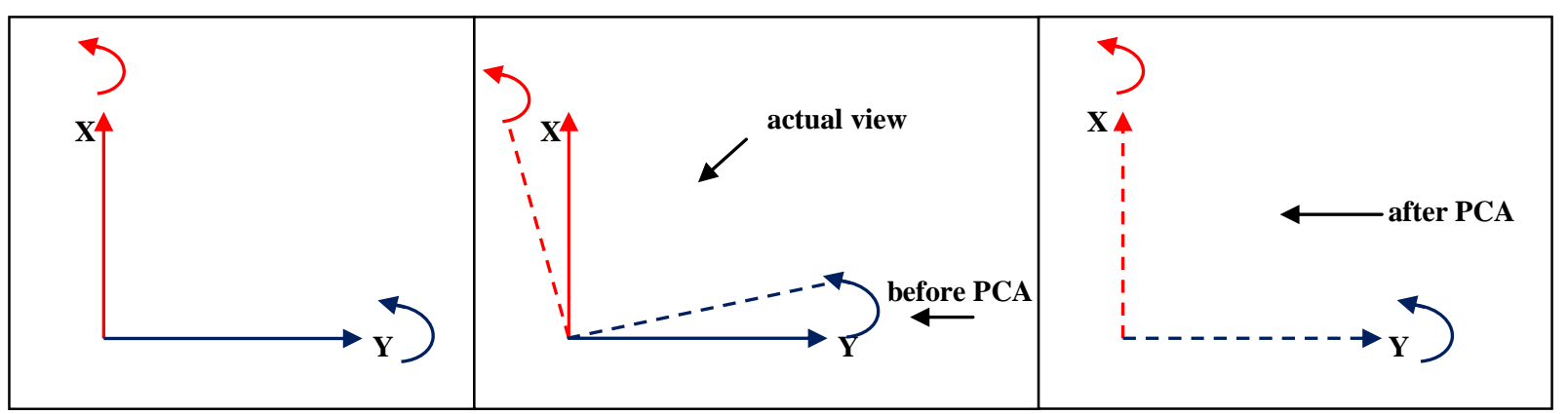

Figure 3. Rotation Perfect arm rotation axis (left), actual view \& view from data (center), actual arm rotation axis rotated by PCA (right). 


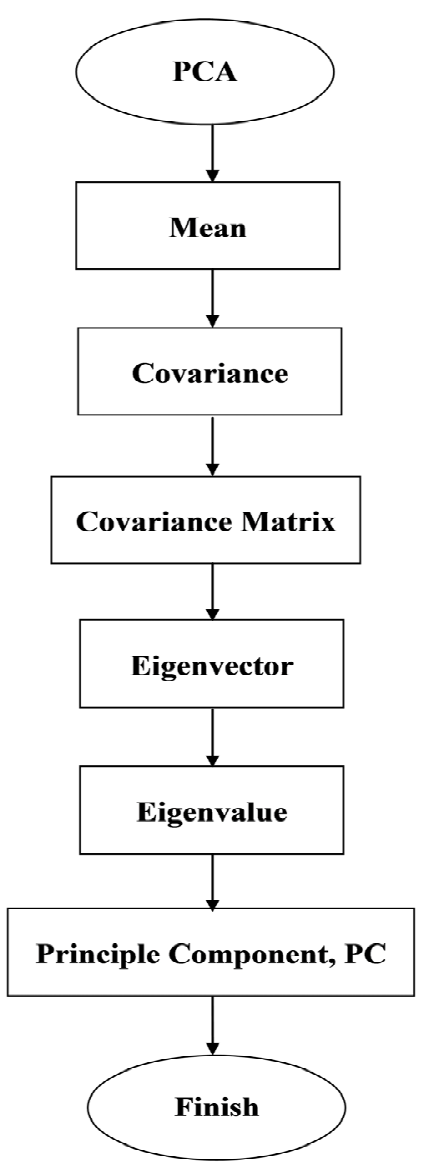

Figure 4. Principle of Component Analysis.

\subsection{Angle of Leaping Motion}

Based on Tom F. Novacheck [6], in a complete cycle of running or jogging motions, the runner will be airborne twice, means that in running activity, there will be at any instance where the test subject leap in order to get airborne. While in walking activity, there will be only phases, where the test subject in a position called stance, where the test subject weight is supported only by a foot, and another one is when both feet touch the ground.

In short, leaping motion does not exist in walking motion and hence, this clear difference is used as the component to differentiate both walking and jogging motions[7]. The leaping motion was measured using angle of acceleration of the test subject.

Thus the second part of the analysis component is leaping motion after the angular velocity. The leaping motion was measured using quiver function in MATLAB to analyze the angle of leaping and direction of leaping. PCA was not used in this analysis in order to maintain the original ground axis of the subject motion.

\section{Result and Discussion}

Figure 5 and Figure 6 show the best view of gyroscope sensor after PCA variables reduction and direction realignment for walking and jogging activities. The plot shows that jogging data are scattered with a range larger than walking activity. The direction and leaping angle for jogging activity are recorded with a range higher than walking activity. Result shows that jogging activity brings bigger swinging angle and higher leaping distance.

Based on the value of mean of angular velocity and leaping angle of test subject's arm, a range is generated for both walking and jogging activities as shown in Table 1 .

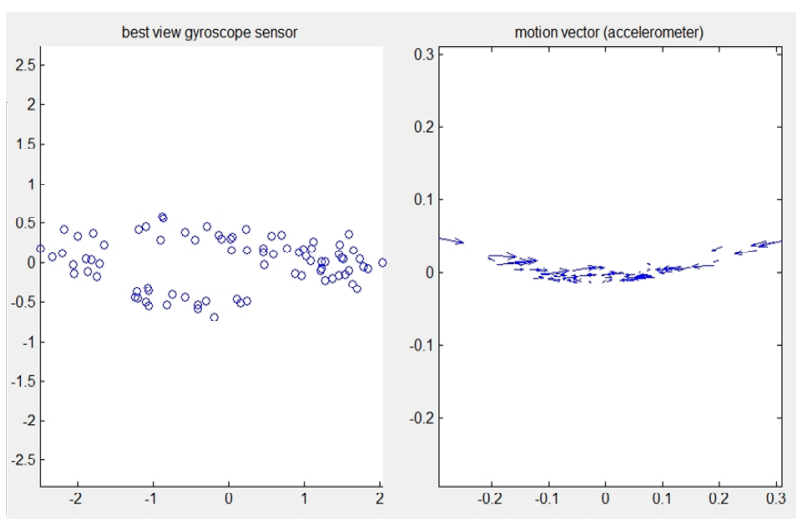

Figure 5. Scatter plot of acceleration of walking motion (left), vector plot of acceleration of walking motion (right).

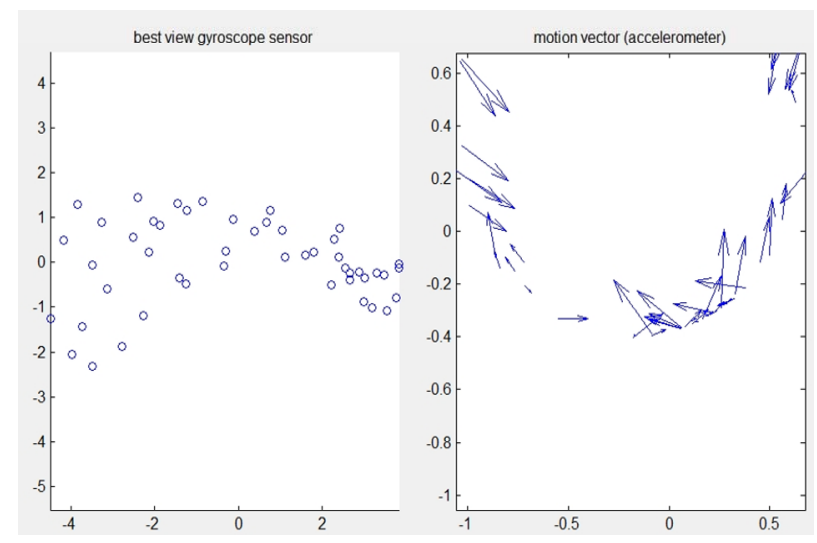

Figure 6. Scatter plot of acceleration of jogging motion (left), vector plot of acceleration of jogging motion (right).

Table 1. range of angular velocity and leaping angle for walking and jogging motion.

\begin{tabular}{ccc}
\hline $\begin{array}{c}\text { Angular velocity } \\
(\mathrm{rad} / \mathrm{sec})\end{array}$ & WALK & $1.1007 \leq$ angular velocity $\leq 1.9119$ \\
& JOG & $2.5697 \leq$ angular velocity $\leq 3.9663$ \\
& WALK & $4.629 \leq$ leap angle $\leq 16.10$ \\
$\begin{array}{c}\text { Leaping angle } \\
\left({ }^{\circ}\right)\end{array}$ & JOG & $38.0579 \leq$ leap angle $\leq 62.3121$ \\
\hline
\end{tabular}


Through observation from the table, the angular velocity of test subject's arm is faster in jogging compared to walking. It means that the test subject swing his arm faster in jogging compared in walking. Since the range of angular velocity of walking and jogging are not overlap with each other, this range is used to recognize and differentiate both walking and jogging motion.

Same thing goes to leaping angle assessment. Jogging activity has recorded a range higher than $35^{\circ}$.

The mean of angular velocity and the mean of leap angle of the test data yield from the analysis are used to evaluate the motion classification program represented by flowchart in Figure 7. A system was developed based on the range of angular velocity and leaping angle for motion classification.

\section{Conclusions}

It can be concluded that a 3-space sensor is useful in analyzing human motion since the device consists of three different sensors which give advantage in measure- ing the motion in several aspects simultaneously[8]. The success of the data collecting session also put wearable sensor approach one step forward of the vision based approach in terms of the number of different measurement can be done simultaneously.

There are two kinds of graph generated in analyzing both motions: they are PCA scatter plot and quiver function graph. PCA scatter plot realigned the graph for the best view in angular velocity and reduced nine variables become three for better analysis. The quiver function in MATLAB is used to generate the direction vector plot of the given motion. The vector plot shows the increment and decrement of the motion direction. This information shows clearly every move for a swing of human arm.

Finally, the range of angular velocity generated in Table 1 is used to develop a motion classification system. The system was trained by using the recent collected data and the process of increasing the amount of data is planned to be done in the future in order to increase the accuracy and specificity of the system.

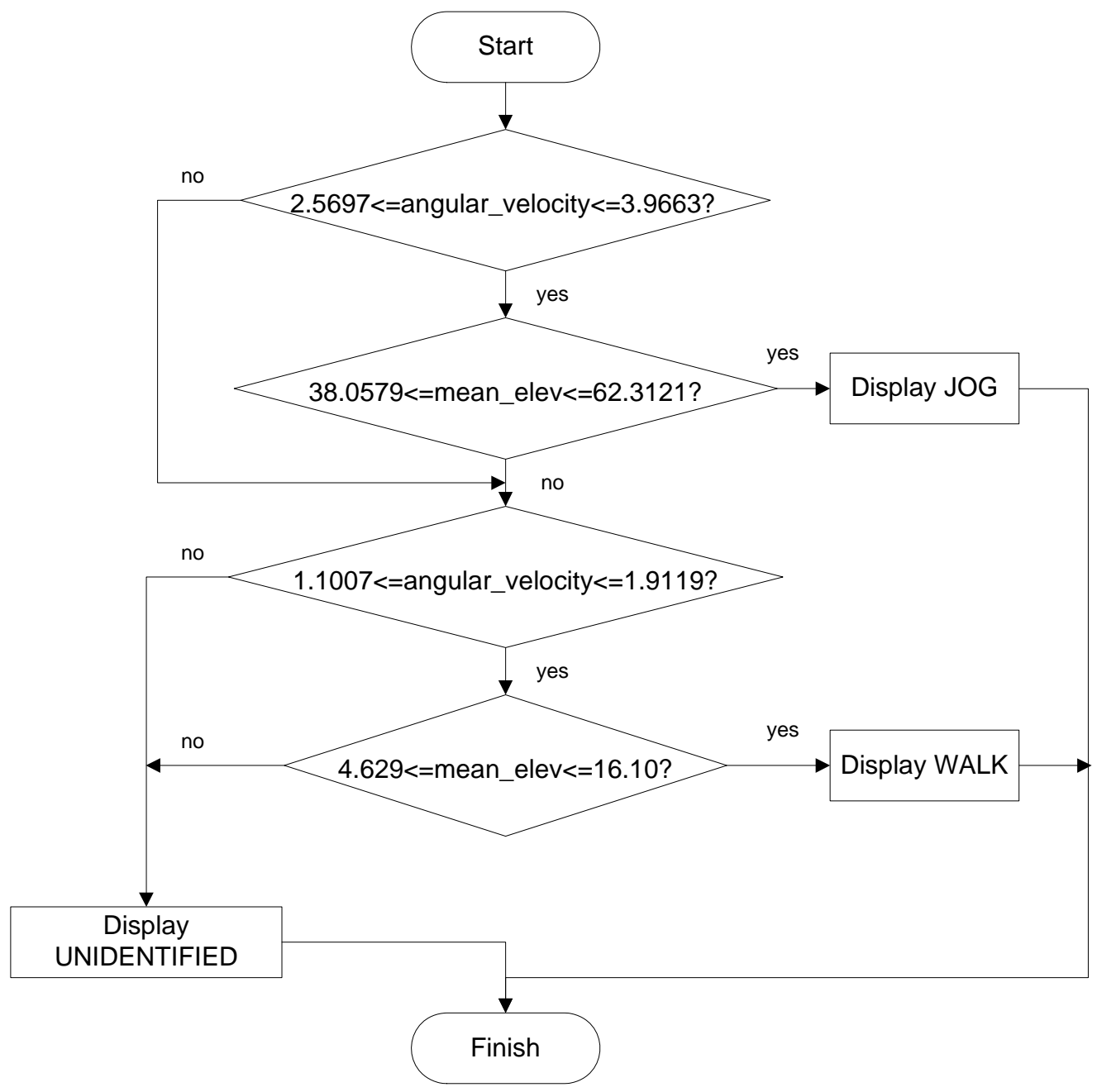

Figure 7. Flowchart of motion classification program. 


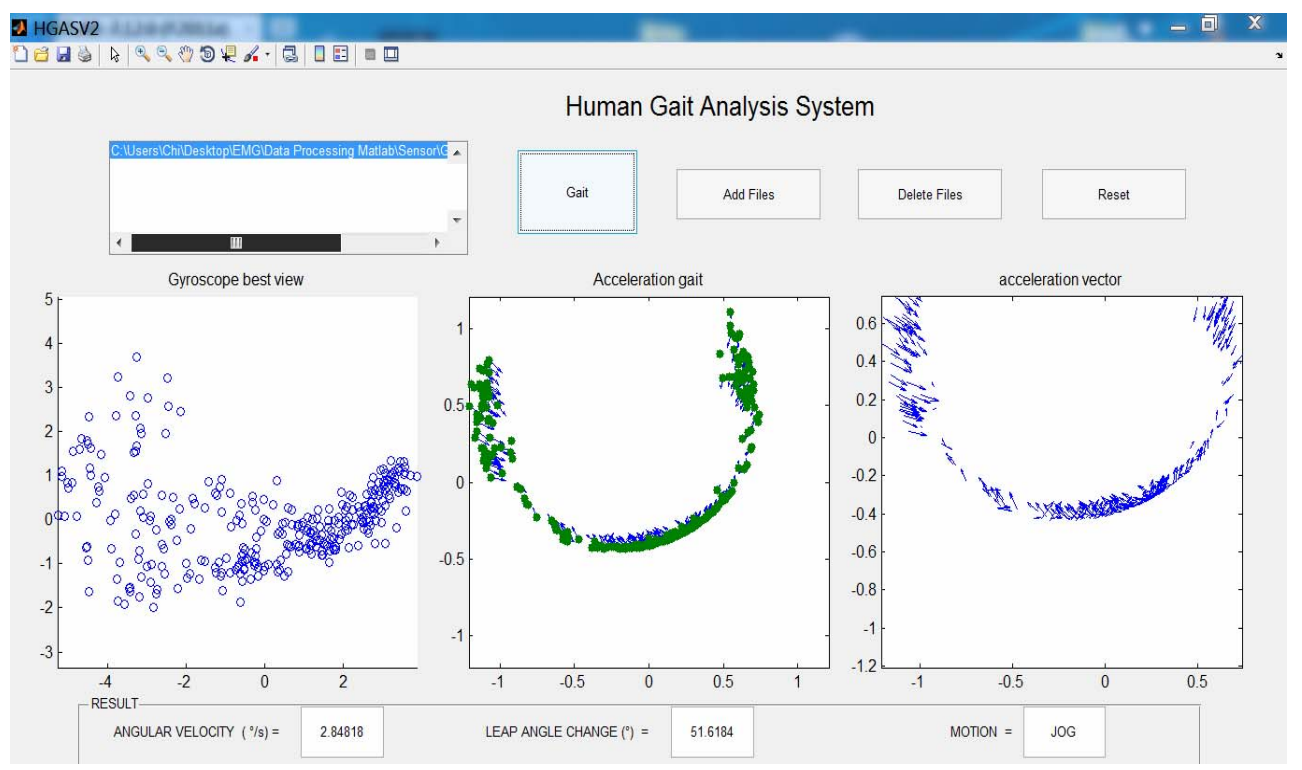

Figure 8. Motion classification system.

The classification system was shown as in Figure 8. The GUI is developed to be user friendly as users can easily load their file to the system within a click. The system displays gyroscope best view plot, acceleration gait plot (human arm swinging animation), and acceleration vector plot (direction of swinging arm). The mean value of angular velocity and mean value of leaping angle of the motion are displayed at the bottom of the plots. The classification result is shown at the bottom right of GUI. The system will display "WALK" for walking activity, "JOG" for jogging activity, and "UNIDENTIFIED” for unidentified motion.

\section{Acknowledgements}

A study of this magnitude depends on the hard work and commitment of many professionals, and we are pleased to acknowledge their contributions. The authors are deeply indebted and would like to express our gratitude to the Universiti Teknologi Malaysia for supporting and funding this study under Research University Grant (QJ13000.2636.05J69) and MyPhD Scholarship Scheme from Ministry of Higher Education (MOHE). Appreciation also goes to Muhammad N. Hafizuddin Zainudin in assisting the data acquisition.

\section{REFERENCES}

[1] L. Tao, I. Yoshio, S. Kyoko and M. Haruhiko, "Development of Wearable Sensor Combinations for Human Lower Extremity Motion," Proceedings 2006 IEEE International Conference on analysis in Robotics and Automation, ICRA 2006.

[2] H. Zhou, T. Stone, H. S. Hu and N. Harris, "Use of Multiple Wearable Inertial Sensors in Upper Limb Motion Tracking," Medical Engineering \& amp; Physics. Vol. 30, No. 1, 2008, pp. 123-133.

[3] R. Liu, J. Z. Zhou, M. Liu and X. F. Hou, "A Wearable Acceleration Sensor System for Gait Recognition," Proceedings of the 2nd IEEE Conference in Industrial Electronics and Applications, 2007.

[4] 3-Space Sensor, User Manual, YEI Technology.

[5] J. Shlens, "A tutorial on Principal Components Analysis, Version 2," Salk institute for Biological Studies and Institute for Nonlinear Science, University of California, San Diego, 2005.

[6] T. F. Novacheck, "The Biomechanics of Running," Gait \& amp; Posture, Vol. 7, No. 1, 1998, pp. 77-95. doi:10.1016/S0966-6362(97)00038-6

[7] M. Hanlon and R. Anderson, "Real-time gait event detection using wearable sensors," Gait \& amp; Posture, Vol. 30, No. 4, 2009, pp. 523-527. doi:10.1016/j.gaitpost.2009.07.128

[8] A. Minami, H. Teruo, O. Tomoyuki, K. Kazuyuki, W. Kajiro and K. Yosuke, "A Study on Gait Analysis by Measuring Axis Rotation Based on 3D Magnetic and Acceleration Sensors," Proceedings of SICE Annual Conference 2010. 